\title{
Las Tecnologías de la Información y de la Comunicación en las Facultades de Educación
}

\author{
José Francisco DurÁn MEDINA \\ Universidad de Castilla La Mancha (España) \\ JoseFrancisco.Duran@uclm.es

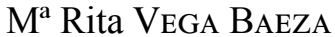 \\ Universidad Autónoma de Zacatecas (México) \\ MRVBaeza@hotmail.com
}

\begin{abstract}
Resumen:
En este artículo se destaca la importancia de una formación inicial docente de calidad en lo que se refiere a las Tecnologías de la Información y de la Comunicación (TIC). Formación Inicial que debe ser incluida como asignatura obligatoria en las Facultades de Educación y que, además, debe completarse indiscutiblemente con una imprescindible formación permanente del profesorado en activo. Esta premisa constituye uno de los pilares fundamentales para conseguir una Educación de calidad, una Educación competente presente y de futuro.
\end{abstract}

Palabras Clave: TIC, Formación, Educación.

\section{The Information and Communication Technologies in the Faculties of Education}

\begin{abstract}
This article highlights the importance of a quality initial teacher formation about the Information and Communication Technologies (ICT). Initial training that it should be included as a compulsory subject in the Faculties of Education and also it should be completed with an imperative continuing education for active teachers. This premise is one of the pillars basic to get a quality education a competent education present and future.
\end{abstract}

Key Words: ICT, Formation, Education.

\section{Referencia normalizada:}

Durán Medina, J. F.; Vega Baeza, M. R. (2013) Las Tecnologías de la Información de la Comunicación en las Facultades de Educación. Historia y Comunicación Social. Vol. 18 № Especial Noviembre. Págs. 313-326.

Sumario: 1. Introducción. Estado de la Cuestión. 2. Metodología. 3. Apartados. 4. Conclusión. 5. Referencias Bibliográficas. 6. Notas. 


\section{Introducción y estado de la cuestión}

La LOGSE (Ley Orgánica General del Sistema Educativo, de 3 de octubre de 1990) establecía las siguientes especialidades en Magisterio: Educación Infantil, Educación Primaria, Educación Musical, Educación Física, Educación Especial (Pedagogía Terapéutica), Audición y Lenguaje, Lengua Extranjera.

Nueve años más tarde, en 1999, tuvo lugar la Declaración de Bolonia (más conocido como Plan Bolonia), acuerdo firmado por ministros de Educación de varios países de Europa en la ciudad de Bolonia, de donde toma su nombre. Mediante este acuerdo se llevarían a cabo intercambios de titulados y se adaptarían los contenidos de las áreas y materias universitarias a las demandas sociales.

Lógicamente, estando en las puertas del Siglo XXI, con las Tecnologías de la Información y de la Comunicación (TIC) en pleno apogeo, provocando una nueva era tan poderosa o incluso más que cualquier otra era anterior (1787-1845: era del vapor; 1845-1895: era de la mecánica; 1895-1947: era de la electricidad; 1947-2003: era de la electrónica; 2003-actualidad: era de la información), resultaría evidente la incorporación inmediata y obligatoria de alguna asignatura al respecto, incluso área entera, dedicada a su conocimiento, desarrollo y aplicación en todos los campos. Y decimos bien "resultaría", tiempo condicional y no imperativo, ni tan siquiera presente o futuro. Desgraciadamente, y desconociendo las razones o motivos por los que se ha llegado a esta situación, no llegó a producirse de manera generalizada esa necesaria adaptación de los contenidos a las demandas sociales que se predicaba y figuraba entre los objetivos del Plan Bolonia.

En nuestro caso concreto, especificando en lo que se refiere a las instituciones dedicadas a la formación de futuros maestros, las diplomatutas existentes hasta entonces pasaron a convertirse en Grados de cuatro años. Hasta ahí, perfecto. Por fin se empezaba a otorgar el valor e importancia que tiene la educación de los futuros ciudadanos, se atisbaba un ligero reconocimiento a la enorme responsabilidad que supone formar integralmente a quienes seguirán construyendo, moldeando y representando a nuestra nación.

Pero no todo podían ser buenas noticias, no. Desafortunadamente (haciendo hincapié en el significado literal de tal vocablo), desaparecen del Currículum Educativo de las Escuelas de Magisterio, ahora Facultades de Educación, piezas tan fundamentales como la asignatura "Nuevas Tecnologías Aplicadas a la Educación", una de las que alcanzaban mayor puntuación por parte de los estudiantes según arrojan las estadísticas extraídas de las encuestas de valoración realizadas anualmente. Y si alguno de ustedes piensa que únicamente se ha modificado el nombre de la asignatura intentando actualizarlo, se equivoca. Simplemente desaparece. Y ya está. Bueno, para ser exactos, se elimina como asignatura obligatoria porque se estima que debería ser impartida como área transversal, en todas las asignaturas del Grado. Y no les falta razón: debería, nuevamente condicional. Pero..., ¿cuál es la condición? ¿El libre 
albedrío del profesorado? Por tanto podrían perfectamente pasarse las TIC por alto y no ser mencionadas en muchas asignaturas, ¿no es así?

Los cambios que sí se han adoptado ya en todo nuestro territorio con la adaptación de carreras universitarias europeas al Plan Bolonia es que estas anteriores diplomaturas de Magisterio desaparecen y se sustituyen por dos Grados de cuatro años: Grado de Maestro/a en Educación Primaria y Grado de Maestro en Educación Infantil.

En los dos últimos años de carrera se pueden cursar algunas asignaturas optativas que, si pertenecen todas al mismo ámbito, llegan a cualificar al estudiante en alguna especialidad. Las Menciones (o especialidades) generalmente ofertadas son: Educación Física, Lengua Extranjera, Educación Musical, Educación Especial y Religión.

\section{Metodología}

La metodología utilizada en este estudio está basada obviamente en técnicas cualitativas de investigación social, en este caso concreto mediante una metodología documental, exploratoria, descriptiva e incluso con ligeros tintes de investigación histórico longitudinal.

Como en toda investigación social basada en documentos, se han reunido, seleccionado y analizado los datos producidos por la sociedad para estudiar el fenómeno determinado de la formación inicial docente en Tecnologías de la Información y de la Comunicación.

Se trata de una investigación exploratoria ya que pretende una visión general, de tipo aproximativo, respecto a la realidad que se presenta: la escasa importancia que se otorga a la necesidad de dominar las nuevas tecnologías por parte del profesorado actual y futuro, tema poco explorado y reconocido hasta el momento debido a su novedad, pero sin duda alguna considerado crucial por los profesionales de la Educación.

También se puede decir que este trabajo hace uso de la metodología descriptiva, explicando minuciosamente la situación actual en cuanto al tema que nos ocupa, especificando los diferentes aspectos, dimensiones y componentes del fenómeno estudiado.

Por último, y reconociendo que un estudio longitudinal es un tipo de estudio observacional que investiga al mismo grupo de personas de manera repetida a lo largo de un período de tiempo (años, en ocasiones décadas o incluso siglos), el estudio sobre la incorporación de algún tipo de asignatura relacionada con las Tecnologías de la Información y de la Comunicación en las Facultades de Educación podría igualmente incluirse en este ámbito porque, a pesar de no trabajar con el mismo grupo de personas, sí se trata de grupos con gran afinidad estudiados en diferentes momentos de nuestra historia próxima. 
En cuanto al muestreo se refiere, atendiendo a los conceptos básicos que lo componen, concretaremos sus elementos:

- Universo: refiriéndonos a los elementos que comparten unas características definidas relacionadas con el problema de investigación, en nuestro caso serían todas las Facultades de Educación del mundo o instituciones educativas semejantes.

- Población: entendida como un conjunto definido, limitado y accesible del universo que forma el referente para la elección de la muestra, sería el grupo al que se intenta generalizar los resultados, es decir, las Facultades de Educación de España.

- Muestra: es el conjunto de individuos extraído de la población a partir de algún procedimiento específico como en este trabajo puede ser la oportunidad o cercanía con la Facultad de Educación de Toledo.

- Elemento o Individuo: siendo la unidad más pequeña en la que podemos descomponer la muestra, la población o el universo, y pudiendo ser una persona, un grupo, un centro, etc., en nuestro caso concreto lo formarían los alumnos de la Mención TICE de la Facultad de Educación de Toledo.

Así, podemos deducir lógicamente las técnicas empleadas durante la realización de este trabajo, que serán no probabilísticas:

- Técnica deliberada, consistente en seleccionar como muestra a algunos sujetos que poseen las características necesarias para la investigación.

- Técnica accidental o casual, seleccionando individuos que casualmente se encuentran en el lugar y en el momento decidido por el investigador.

Problema que todo esto conlleva: la dificultad de generalización de resultados.

\section{Situación actual}

\subsection{Visión general en las universidades españolas}

Volvamos un momento al punto donde nos habíamos quedado en el primer apartado, cuando se había expuesto la desaparición de la única asignatura relacionada con las Nuevas Tecnologías de la Información y de la Comunicación aplicadas a la Educación, cuando se intuía que la formación de los futuros maestros (sin descartar a los presentes) se dejaría en manos del libre albedrío del profesorado universitario, tanto si se encontraban entre los denominados tecnófilos como entre los tecnófobos.

En esta tesitura no nos debe extrañar que la respuesta no haya sido unívoca, existiendo en la actualidad diversidad de formas o maneras que las distintas instituciones educativas han considerado oportunas para que tan importante y necesario conoci- 
miento no termine perdiéndose y desapareciendo entre las oscuras nubes del saber general.

Algunas facultades de Educación en España (aunque muchas menos de las deseables) ofertan asignaturas optativas que cualifican para obtener una Mención específicamente relacionada con las TIC. Algunos buenos ejemplos son los siguientes:

- Facultad de Educación de la Universidad Autónoma de Madrid: Mención TIC.

- Facultad de Ciencias de la Educación, Enfermería y Fisioterapia de la Universidad de Almería: Mención en TIC y Comunicación en el Aula.

- Facultad de Educación de la Universidad de Cantabria: Mención Las TIC en el contexto del Centro Escolar: integración, organización e interactividad.

- Facultad de Ciencias de la Educación de Santiago de la Universidad de Santiago de Compostela: Mención en Educación y TIC.

- Universidad Internacional de Valencia (Online): Mención en TIC en Educación.

- Facultad de Humanidades y Ciencias Sociales de la Universidad Internacional Isabel I de Castilla (Online): Mención en Educación y TIC.

- Facultad de Educación de Toledo de la Universidad de Castilla La Mancha: Mención TICE Tecnologías de la Información y de la Comunicación en Educación.

Intentemos ahora conocer más en profundidad los detalles de la Mención TICE de la Facultad de Educación de Toledo, en la universidad de Castilla La Mancha.

\subsection{Facultad de Educación De Toledo. UCLM}

La Mención TICE en la Facultad de Educación de Toledo (Universidad de Castilla La Mancha - España) comenzó su aventura en el Curso 2011-2013, gracias al esfuerzo y afán constructivo del equipo de docentes del Departamento de Pedagogía, quienes liderados y alentados por el Profesor Doctor Ricardo Fernández Muñoz (buen tecnófilo, excelente educador y mejor comunicador), consiguieron convencer al claustro de profesores de la facultad sobre la importancia y necesidad de esta formación en los futuros maestros.

Los treinta créditos necesarios para la consecución de la mención TICE en Toledo, se reparten entre cinco asignaturas de seis créditos cada una, dos de ellas incorporadas al tercer curso del Grado en Magisterio en cualquiera de sus dos especialidades (Infantil o Primaria), y las otras tres asignaturas incorporadas en el cuarto curso del Grado en Magisterio.

\subsubsection{Tercero Curso de Grado en Magisterio}


En el Tercer Curso para obtener el Título de Graduado en Magisterio, en cualquiera de sus dos especialidades (Educación Infantil y Educación Primaria) se pueden cursar las dos siguientes asignaturas relacionadas con las TIC que posibilitan la consecución de la Mención TICE:

\subsubsection{Optativa 1 / Mención TICE: Cultura y Pedagogía Audiovisual}

Impartida por dos profesores mediante la coordinación de los departamentos de Expresión Plástica y Pedagogía, pretende que su alumnado adquiera las siguientes competencias:

- favorecer la alfabetización audiovisual para el desarrollo del rol profesional de los futuros maestros.

- Promover la creación de nuevas formas de expresión mediante la explotación didáctica de recursos tecnológicos variados.

Esta asignatura se estructura en cuatro grandes módulos de contenido:

- Alfabetización visual. Hacia una pedagogía de la imagen.

- Los medios audiovisuales (MAV) en la escuela.

- Medios de imagen fija y sonido en la Enseñanza.

- Medios de imagen móvil y Enseñanza.

Conscientes de la importancia de experiencias prácticas que faciliten la comprensión de los contenidos teóricos, la Facultad de Educación de Toledo completa el estudio de estas asignaturas con otras actividades extracurriculares tan importantes (incluso a veces más) como las incluidas en las guías didácticas. En este caso concreto, en la asignatura "Cultura y Pedagogía Audiovisual", las actividades que se llevan a cabo son:

- Experiencias reales con medios de comunicación: televisión, radio, prensa...

- Edición de Revista Digital.

- Videoconferencias, donde el alumnado puede participar como ponentes e impartir seminarios a otras universidades internacionales (El vídeo educativo, La fotografía en la escuela...).

- Concursos fotográficos.

3.2.1.2. Optativa 2 / Mención TICE: Las TIC Aplicadas a la Educación y la Didáctica de las Ciencias Experimentales

En este caso, un profesor del Departamento de Pedagogía especialista en Didáctica de las Ciencias Experimentales intenta que el alumnado sea competente para...:

- Conocer los contenidos básicos del currículo de Educación Infantil y Primaria en relación con las Ciencias Experimentales. 
- Utilizar distintos recursos relacionados con las TICE de cara a la enseñanza-aprendizaje de las Ciencias de la Naturaleza en la escuela.

- Aprovechar las posibilidades didácticas de cada recurso relacionado con las TICE y la información que puede proporcionar para la enseñanza-aprendizaje de las Ciencias de la Naturaleza en cada etapa escolar.

- Diseñar y elaborar recursos y materiales concretos y adecuados a partir de las TICE para facilitar la enseñanza-aprendizaje de las Ciencias de la Naturaleza en la escuela.

Tres son sus módulos de contenido:

- Las Ciencias Experimentales en la Educación Infantil y Primaria.

- Integración curricular de las Tecnologías de la Información y de la Comunicación en la Didáctica de las Ciencias Experimentales para Educación.

- Diseño y elaboración de recursos tecnológicos para la Didáctica de las Ciencias Experimentales.

La experiencia práctica en esta ocasión se basa principalmente, y como no podía ser de otra manera, en salidas a la Naturaleza para trabajar directamente con lo visto durante el curso.

\subsubsection{Cuarto Curso de Grado en Magisterio}

Y en el cuarto y último curso para la obtención del título de Graduado en Magisterio se sitúan estas otras tres asignaturas de la Mención TICE que se exponen a continuación:

3.2.2.1. Optativa 3 / Mención TICE: Organización y Gestión de las TIC en los Centros Educativos

Exclusiva del Departamento de Pedagogía, con las siguientes competencias específicas:

- Las TIC en la organización del centro y la comunicación con el entorno.

- La utilización de las TIC en la práctica docente de aula.

- Los usos de las TIC por parte del alumnado.

- La Valoración y expectativas sobre el impacto del Programa Escuela 2.0 o similares a nivel nacional y regional.

Se estructura en tres módulos de contenido:

- Organización de los recursos tecnológicos en los Centros Educativos. El coordinador TIC, funciones y competencias. 
- Proyectos y programas para la gestión de Centros Educativos mediante las TIC (Papás, Delphos,...). La Escuela 2.0: concepción y líneas de acción futura.

- Experiencias y buenas prácticas en la gestión de las TIC en los Centros Escolares.

Para completar la asignatura se realizan actividades prácticas, como la participación en un Ciclo de Conferencias TICE, donde diversos ponentes exponen buenas experiencias relacionadas con la temática: antiguos alumnos emprendedores trabajadores autónomos, directores de Centros Públicos que utilizan las TIC en su labor cotidiana de Organización y Gestión del colegio que dirigen, etc.

\subsubsection{Optativa 4 / Mención TICE: Diseño y desarrollo de recursos tecnológicos} educativos

Igualmente dependiendo del Departamento de Pedagogía, su objetivo principal es que el alumnado sea competente para...

- Conocer la existencia de diferentes recursos tecnológicos educativos y sus tipologías, desarrollando una clasificación propia actualizada.

- Describir, analizar y utilizar los recursos tecnológicos habituales de los Centros Educativos, mostrando sus ventajas y desventajas.

- Diseñar y crear recursos tecnológicos educativos propios.

Para ello, en consecuencia, plantea la asignatura distribuyéndola y estructurándola en los siguientes tres módulos de contenido:

- Clasificación/Tipología de los recursos tecnológicos en los Centros Educativos.

- Descripción de recursos tecnológicos educativos. Utilización en los Centros Educativos.

- Diseño y creación de recursos tecnológicos educativos propios.

Y como experiencias prácticas de aprendizaje, además de continuar con el Ciclo de Conferencias TICE (aunque en este caso concretando en la utilización por parte del alumnado de colegios públicos de Educación Infantil y Primaria, incluso con conferencias impartidas por alumnado de quinto y sexto de Primaria), también se continúan las videoconferencias internacionales con otros profesores y alumnos de distintas universidades: Chile, Colombia, Venezuela, México, España...

\subsubsection{Optativa 5 / Mención TICE: Comunidades Virtuales y Redes Sociales en} Educación

En este último caso, son dos los departamentos que se encargan de su docencia, Pedagogía y Sociología, y persiguen alcanzar las siguientes competencias específicas durante su desarrollo en el curso escolar: 
- Analizar las posibilidades y limitaciones que ofrecen las redes sociales en el desarrollo de proyectos de enseñanza- aprendizaje a través de TIC.

- Conocer las redes y los recursos fundamentales para la docencia.

- Crear, utilizar e integrar redes-entornos personales de aprendizaje para el desarrollo personal y futuro profesional.

Estos tres objetivos generales se intentarán alcanzar mediante la adquisición de los contenidos repartidos en tres módulos:

- Conectivismo y aprendizaje en red. Utilización de redes en el contexto escolar. Redes sociales y menores: valoración crítica e implicaciones educativas.

- Redes profesionales de aprendizaje para futuros docentes. Trabajo colaborativo en espacios virtuales. Comunidades de aprendizaje en red.

- Experiencias y buenas prácticas educativas en red.

Del mismo modo que las anteriores asignaturas de la Mención TICE impartidas en la Facultad de Educación de Toledo, en la Universidad de Castilla La Mancha (España), ésta última también ofrece un Ciclo de Conferencias con la temática correspondiente, debates internacionales y, lógicamente, experiencias prácticas en las redes sociales, como pueden ser:

Experiencias en Red: Facebook Facultad de Educación de Toledo: https://www. facebook.com/educaciontoledoUCLM

Herramienta social que pone en contacto a la gente de la Facultad de Educación de Toledo con sus amigos y con otras personas que trabajan, estudian y viven en su entorno.

Experiencias en Red: Facebook Mención TICE Facultad de Educación de Toledo: https://www.facebook.com/groups/TICE.Toledo

Herramienta social que pone en contacto a la gente relacionada con la Mención TICE de la Facultad de Educación de Toledo con sus amigos y con otras personas que trabajan, estudian y viven en su entorno.

Experiencias en Red: Pinterest Magisterio Toledo: http://pinterest.com/Magisteriouclm/

Red social para compartir imágenes tomadas en Magisterio de Toledo, que permite a los usuarios crear y administrar, en tableros personales temáticos, colecciones de imágenes como eventos, intereses, hobbies...

Experiencias en Red: Pinterest Magisterio Toledo Mención TICE: http://pinterest. com/magisterioto/tice/

Red social para compartir imágenes tomadas por personal relacionado con la Mención TICE de Magisterio de Toledo, que permite a los usuarios crear y admi- 
nistrar, en tableros personales temáticos, colecciones de imágenes como eventos, intereses, hobbies...

Experiencias en Red: Twitter Alumnos TICE Universidad de Castilla La Mancha: https://twitter.com/TICE_UCLM

Red que permite a los alumnos y profesores de la Mención TICE de la Universidad de Castilla La Mancha enviar mensajes de texto plano de corta longitud, con un máximo de 140 caracteres, llamados tweets, que se muestran en la página principal del usuario. Los usuarios pueden suscribirse a los tuits de otros usuarios, lo que se llama "seguir", y a los usuarios abonados se les llama "seguidores", "followers" y a veces tweeps.

Experiencias en Red: Scoop it Mención TICE Facultad de Educación de Toledo: http://www.scoop.it/t/tice-tecnologias-de-la-informacion-y-la-comunicacion-en-educacion

Se trata de una nueva forma de reunir información para mostrarla y compartirla de manera atractiva en Internet. Es un sistema que permite compartir contenido estimado útil en la red por las personas relacionadas con la Mención TICE de la Facultad de Educación de Toledo. El término que se emplea para definir esta actividad es el de "curación de contenidos", y consiste en el proceso de filtrar, agrupar y compartir toda la información sobre un tema.

Blog difusión de los trabajos de alumnos de la Mención TICE: http://magisterioto-trabajos.blogspot.com.es/p/portafolios-tice.html

Este blog es un sitio web en el que profesores y alumnos de la Mención TICE de la Universidad de Castilla La Mancha publican artículos, y donde suele ser habitual que los propios lectores participen activamente a través de sus comentarios. Sirve para publicar ideas propias y opiniones sobre diversos temas. En este caso concreto, se utiliza sobre todo para exponer los trabajos realizados por los alumnos de la Mención TICE

Marcadores Sociales Diigo Mención TICE: https:/groups.diigo.com/group/ tice-facultad-de-educacin-de-toledo

Profesores y alumnos de la Mención Tice que nos ocupa utilizan Diigo como un sistema de gestión de información personal que incluye marcadores web, bloc de notas post-it, archivo de imágenes y documentos, así como selección de textos destacados. Permite la creación de grupos (públicos o privados) para compartir enlaces favoritos.

Redes profesionales: Linkedin UCLM TIC: http://www.linkedin.com/groups/ TICE-formaci\%C3\%B3n-futuros-maestros-UCLM-4060037.S.226226425?qid=c7937a9a-5450-4c4c-b732-b2535811d298\&goback=.gmp_4060037

Uno de los propósitos de este sitio es que los profesores y alumnos de la Mención Tice de la Facultad de Educación de Toledo puedan mantener una lista con información de contactos de las personas con quienes tienen algún nivel de relación, llamado 
Conexión. Los usuarios pueden invitar a cualquier persona (ya sea un usuario del sitio o no) para unirse a dicha conexión.

Experiencias en Red: Canal en Youtube de la Mención TICE: http://www.youtube. com/playlist?list $=$ PL07A45539A71FBA5F

Espacio web desde el que podrá acceder a una recopilación de los contenidos audiovisuales más relevantes sobre la actividad cultural creados, recomendados $\mathrm{u}$ organizados desde la Mención Tice de la UCLM.

Etc.

De igual modo, cabría destacar que el alumnado que cursa la Mención TICE en la Facultad de Educación de Toledo (España), en Tercero y Cuarto del Grado en Magisterio asiste a otras variadas e importantes actividades formativas que van surgiendo durante los cursos escolares, sin ser particularmente adscritas a ningún curso o asignatura concretos, pero sí que surgen desde la Mención. Algunos de los ejemplos que se podrían considerar de especial relevancia son:

Jornadas Internacionales TIC:

I Jornada TICE. Nuevas Alfabetizaciones. Con la presencia de afamados y expertos autores como Francisco García, Domingo Gallego, Catalina Alonso, Juan Ignacio Aguaded, Manuel Gértrudix...

http://www.ciberimaginario.es/?Itemid=276

La Facultad de Educación de Toledo presentó por primera vez una jornada dedicada a las Tecnologías de la Información y Comunicación en Educación (TICE).

La Jornada examinó los diferentes contenidos y servicios que ofrece la Web 2.0 y las potencialidades tecnológicas de los dispositivos actuales de movilidad, el cómo educar en ellos y qué habilidades y competencias se necesitan para aprender con ellos.

El formato de ponencias y mesas redondas permitió que los ponentes dispusieran de un tiempo suficiente para contar el contexto de la experiencia, sus características, el modo de ponerla en práctica y desarrollarla, y sus principales resultados.

I Jornada PDI. Nuevas perspectivas didácticas y tecnológicas de la Pizarra Digital. De igual manera, con la presencia de notables ponentes: José Dulac, Cristina Alconada, Domingo Gallego, Catalina Alonso, Paulo Nunes...

Se trataron temas de gran interés y actualidad: mesa Redonda sobre "Nuevas perspectivas didácticas y tecnológicas en el aula", presentación del libro "Guía Didáctica para el notebook11", proyectos educativos colaborativos Smartcity EDU, nuevos recursos y tecnologías de Aprendizaje Colaborativo, experiencias con Pizarra Digital de los estudiantes de la mención TICE de la UCLM...

II Jornada Pizarra Digital Interactiva, con la participación de profesores y alumnos de la Facultad de Educación de Toledo, siendo invitados todos los centros educativos 
que imparten enseñanzas en Educación Infantil, Educación Primaria y Educación Secundaria de la provincia de Toledo, tanto públicos, como concertados y privados,

Prácticas TICE en Centros Educativos.

En el tercer curso de todas las especialidades del Grado en Magisterio se cursa el Prácticum I, 18 créditos de prácticas en centros educativos. Durante estas prácticas, los alumnos de la Mención TICE deben colaborar en una investigación a nivel nacional en la que se estudia y valora la utilización de las Tecnologías de la Información y de la Comunicación por parte de los maestros tutores que les corresponden a cada uno de ellos.

Durante el cuarto curso de Grado en Magisterio ocurre otro tanto, pero en este caso son 24 los créditos que se cursan, tiempo suficiente para terminar los trabajos de investigación iniciados el curso anterior y llegar a las correspondientes conclusiones.

Un apartado especial merecen los primeros TFG (Trabajos Fin de Grado) que han ido surgiendo en estos dos primeros cursos de vida de nuestra Mención TICE. En el curso actual ya terminado 2012-2013, tratándose del primer año en el que podía surgir algún TFG ya que la Mención se inició en el curso 2010-2011, se valora como un rotundo éxito la realización de doce Trabajos Fin de Grado incardinados en la Mención TICE y con temáticas concretas específicamente tecnológicas. Se considera conveniente exponer los títulos de estos TFG como prueba de su interés y relación con las TIC:

Diseño e Implementación de un Proyecto Didáctico con integración de las TIC en un aula de Educación Infantil.

Los entornos personales de aprendizaje en la formación de los futuros maestros.

Comunidades virtuales como respuesta a un aprendizaje no formal en la formación permanente del profesorado.

Delphos Papás en la organización del centro y la comunicación con el entorno familiar: análisis de una experiencia.

La pizarra digital interactiva como recurso potenciador de la motivación.

La integración de las TIC en el C.E.I.P. San Antonio de Yeles: un estudio de viabilidad.

Implicaciones educativas de las redes sociales en el C.E.I.P. escultor "Alberto Sánchez".

El uso de la pizarra digital interactiva en el aula de Educación Infantil.

Plataformas virtuales Educativas y su influencia en la metodología docente.

La clase $3^{\circ} \mathrm{C}$ del C.E.I.P. San Juan Evangelista, les presentamos nuestro blog.

Estudio experimental sobre el análisis de una red descriptiva causal para evaluar la influencia de la alfabetización digital en las políticas educativas. 
La mejora de la comprensión lectora mediante las TIC en el Primer Ciclo de Primaria.

Con este plan de estudios estimamos suficiente la preparación y formación en la aplicación de Tecnologías de la Información y de la Comunicación en Educación, si bien hay que señalar que el objetivo primordial de esta mención es, además del lógico y necesario conocimiento de esas TIC así como de la competencia para crear recursos propios, el total convencimiento de la necesidad de un cambio en el paradigma metodológico utilizado en los centros escolares.

\section{Conclusión}

Hace ya más de diez años, los pioneros en el uso de las redes telemáticas en la escuela en nuestro país, decían que la comunicación electrónica, la apertura de la escuela al exterior gracias al correo electrónico y a las redes de telecomunicaciones existentes, representa una auténtica revolución para una escuela que hasta hace poco se encontraba cerrada dentro de las cuatro paredes y con muy poca comunicación hacia fuera (Durán, 2005).

En los últimos años de la primera década y el umbral de la segunda del nuevo siglo, la Unión Europea ha venido manifestando una especial preocupación por la relación de los jóvenes con las TIC y su alfabetización mediática, en especial con las denominadas "pantallas": Internet, televisión, móvil. En ese sentido, la recomendación europea pasa por la recomendación de que cada país ponga un especial empeño en la alfabetización multimedia de su población infantil y juvenil (Ortiz, Rodríguez y Pérez, 2011).

El futuro de las redes y de las empresas de gestión informática aparecerá como más o menos despejado en la medida en que los canales y sistemas físicos sepan llenarse de contenidos y de circulante (Sánchez, 2008).

La presencia cada vez más extendida de los videojuegos en los hogares y en los centros de ocio, su concreción en un 'electrodoméstico' más del entretenimiento en los hogares, ha ido afirmando su importancia en la cultura (popular) y ha facilitado su atención académica desde diferentes áreas del conocimiento: la literatura comparada, el diseño gráfico, la cinematografía, la semiótica o la psicología entre otras. Estas iniciativas asumen una correspondencia directa entre el nuevo campo de estudio y unas metodologías consolidadas que se intentar adaptar al nuevo objeto (Gómez, 2007). 


\section{Referencias bibliográficas}

DURÁN, J. F. (2005). "Herramientas Educativas en Internet". En SEECI: Sociedad Española de Estudios de la Comunicación Iberoamericana, $\mathrm{n}^{\circ}$ 12, Madrid: Universidad Complutense de Madrid. p. 84-107.

GÓMEZ, S. (2007). "Videojuegos: El desafío de un nuevo medio a la Comunicación Social". En HyCS: Historia y Comunicación Social, $\mathrm{n}^{\mathrm{o}}$ 12, Madrid: Universidad Complutense de Madrid. p. 71-82.

ORTIZ, M. A., RODRÍGUEZ, D. y PÉREZ, M. J. (2011). "Perfil de entrada de los futuros alumnos de comunicación en las universidades de Madrid, en 2010/2012: la reafirmación de los rasgos". En SEECI: Sociedad Española de Estudios de la Comunicación Iberoamericana, $\mathrm{n}^{\mathrm{o}}$ 26, Madrid: Universidad Complutense de Madrid. p. 1-21.

SÁNCHEZ, J. R. (2008). "Vectores tecnológicos de la convergencia en el sector Info+Com". En HyCS: Historia y Comunicación Social, nº 13, Madrid: Universidad Complutense de Madrid. p. 155-166.

\section{Los autores:}

José Francisco Durán Medina: Profesor Doctor en la Facultad de Educación de Toledo (Universidad de Castilla La Mancha - España), con numerosos artículos en revistas indexadas, libros, capítulos de libros, participación en congresos internacionales, importantes Proyectos de Investigación Nacionales e Internacionales, etc., con variadas líneas de trabajo en su gran mayoría relacionadas con las TIC aplicadas a la Educación. El Currículum Vitae completo se puede visitar en http://educacion. to.uclm.es/pdf/investigacion/CNV/CVN_francisco-duran.pdf

$\mathrm{M}^{\mathrm{a}}$ Rita Vega Baeza: Poeta. Psicoanalista y doctora en filosofía por la universidad Complutense de Madrid es Catedrática de la Universidad Autónoma de Zacatecas y docente de la maestría en Investigaciones Humanísticas y Educativas de la Unidad Académica de Docencia Superior de dicha Universidad. Tiene numerosas publicaciones de poesía en revistas nacionales y extranjeras. 